\title{
Erratum: Top quark mass dependence of the Higgs boson-gluon form factor at three loops [Phys. Rev. D 100, 034017 (2019)]
}

Joshua Davies, Ramona Gröber, Andreas Maier, Thomas Rauh, and Matthias Steinhauser®

(Received 27 August 2020; published 15 September 2020)

DOI: $10.1103 /$ PhysRevD.102.059901

The three-loop term of Eq. (8) of this paper follows the definition in Ref. [1] where the renormalization scale has been set to $\mu^{2}=\hat{s}$ in the Green function. Converting this to the definition of this paper with $\mu^{2}=-\hat{s}-i 0$, Eq. (8) reads

$$
\begin{aligned}
F_{\triangle}^{(2), \text { fin }} \underset{\underbrace{\rightarrow}}{ } & -\frac{8 \pi^{3}}{27}\left(3+\pi^{2}\right) \sqrt{1-z} \\
& +\frac{\pi^{2}}{54}\left[\left(458-15 \pi^{2}-44 n_{l}+\left(198-12 n_{l}\right)\left(L_{s}-2 \ln 2\right)\right) \log (1-z)\right. \\
& \left.-\left(99-6 n_{l}\right) \log ^{2}(1-z)\right](1-z)+\mathcal{O}\left((1-z)^{3 / 2}\right),
\end{aligned}
$$

with $L_{s}=\log (-4 z-i 0)$. Note that the additional term is proportional to $\left(L_{s}-2 \ln 2\right)=-i \pi+\mathcal{O}(1-z)$ and does not affect the real part of the coefficient.

The different input from the threshold region leads to changes in the Padé approximants entering Figs. 4, 5, and 6 of this paper. We find that it is no longer required to enhance the exclusion region for the coefficient of $L_{s}$ for $F_{\Delta}^{(2,0) \text {, fin }}(z)$ to Eq. (14) in this paper. On the other hand, we needed to split the non- $L_{s}$ term $F_{\Delta}^{(2) \text {,fin }}(z)$ into the $n_{h}$ contribution with an additional heavy-quark loop and the remainder. For the $n_{h}$ term we relaxed the exclusion region to

$$
\operatorname{Re}\left(z\left(\omega_{0}\right)\right) \geq-1 \quad \text { and } \quad\left|\omega_{0}\right| \leq 1.2
$$

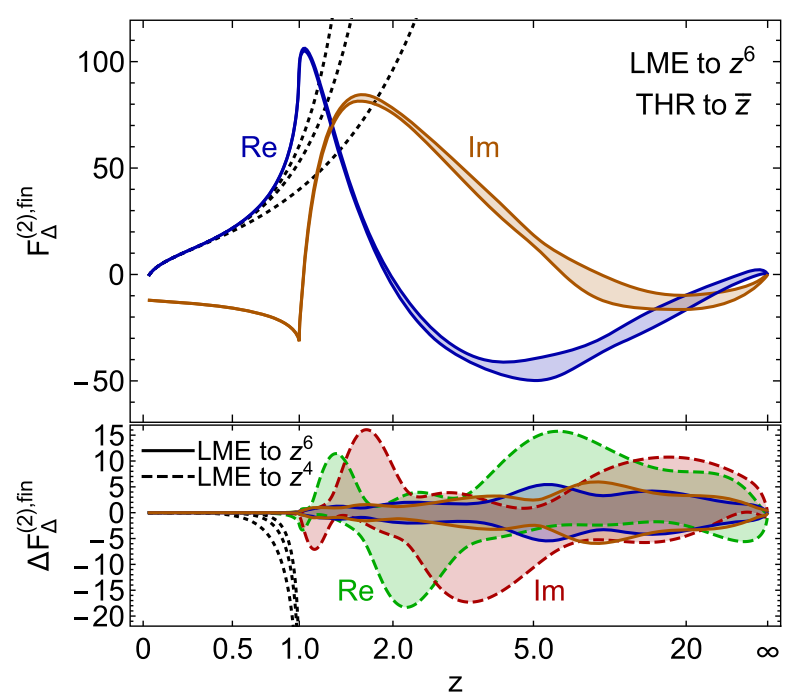

FIG. 1. Our approximations for the real and imaginary parts of the three-loop triangle form factor are shown in blue and orange, respectively. The bands give the standard deviation of the considered set of Padé approximants. The dashed lines correspond to the real part up to order $z^{2}, z^{4}$, and $z^{6}$. The lower panel shows the differences to the central values; see text for details.

Published by the American Physical Society under the terms of the Creative Commons Attribution 4.0 International license. Further distribution of this work must maintain attribution to the author(s) and the published articles title, journal citation, and DOI. 

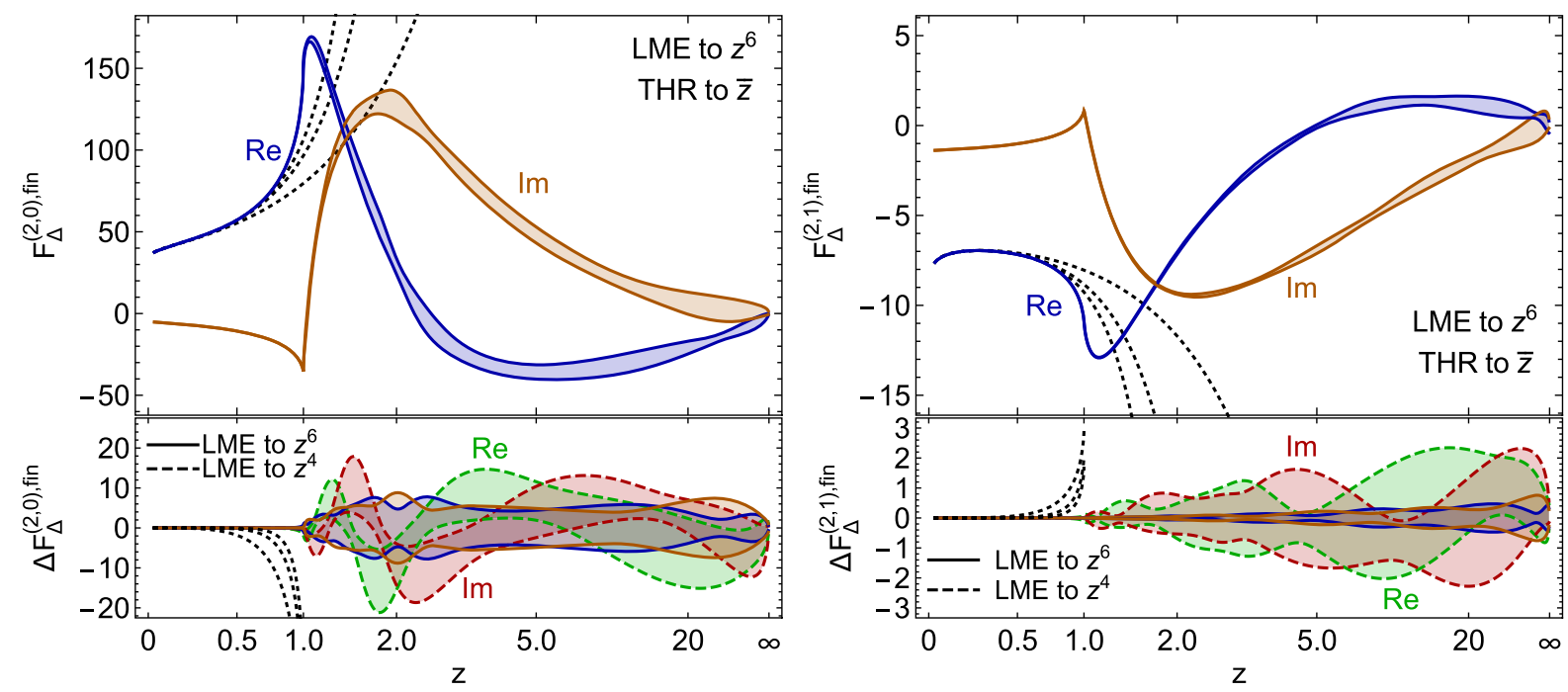

FIG. 2. Our approximations for the three-loop form factor separated according to the light-fermion contributions.

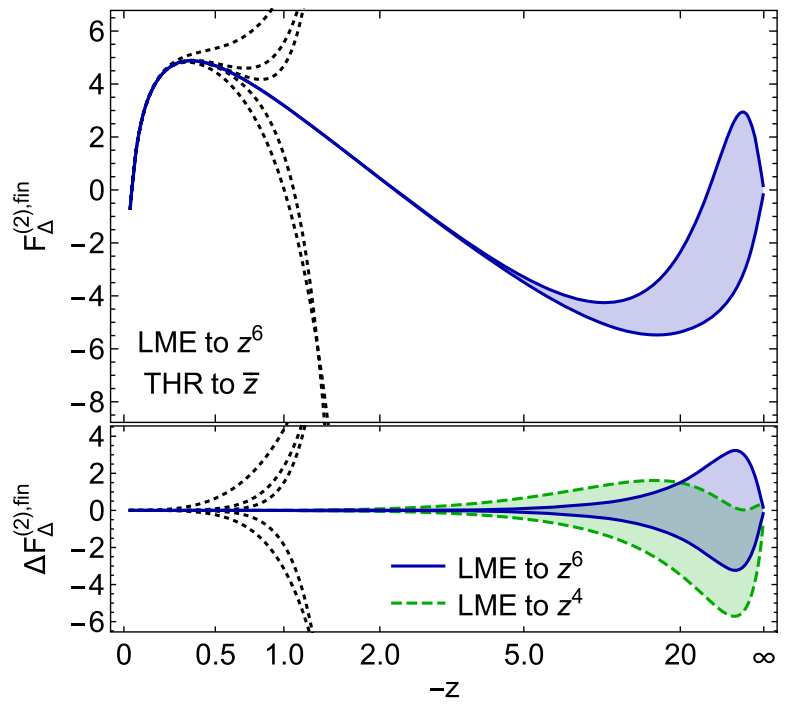

FIG. 3. The three-loop result for the form factor $F_{\triangle}^{(2), \text { fin }}$ for spacelike momenta. The dashed lines correspond to the real part of the LME approximation up to order $z^{2}, z^{3}, z^{4}, z^{5}$, and $z^{6}$. The same notation as in Fig. 2 is adopted.

to find a sufficient number of Padé approximants. The residues of the poles in the region $-2 \leq \operatorname{Re}\left(z\left(\omega_{0}\right)\right) \leq-1$ are typically three orders of magnitude smaller than those in the remaining part of the complex plane and therefore do not cause any visible unphysical resonances. The updated plots are shown in Figs. 1, 2, and 3, respectively.

The sentence in the second-to-last paragraph of Sec. III "For $|z|>20$ the Padé approximation procedure does not lead to stable results which is also seen by the fact that the uncertainty becomes larger after incorporating more expansion terms (see lower panel)." should now read "For $|z|>20$ the Padé approximation procedure does not lead to accurate results, we note that incorporating additional expansion terms does not significantly affect the size of the uncertainty.".

Note that there is no qualitative change of the result and thus the conclusions are not affected. We have updated the results in Ref. [2].

[1] R. Gröber, A. Maier, and T. Rauh, J. High Energy Phys. 03 (2018) 020.

[2] https://www.ttp.kit.edu/preprints/2019/ttp19-014/. 